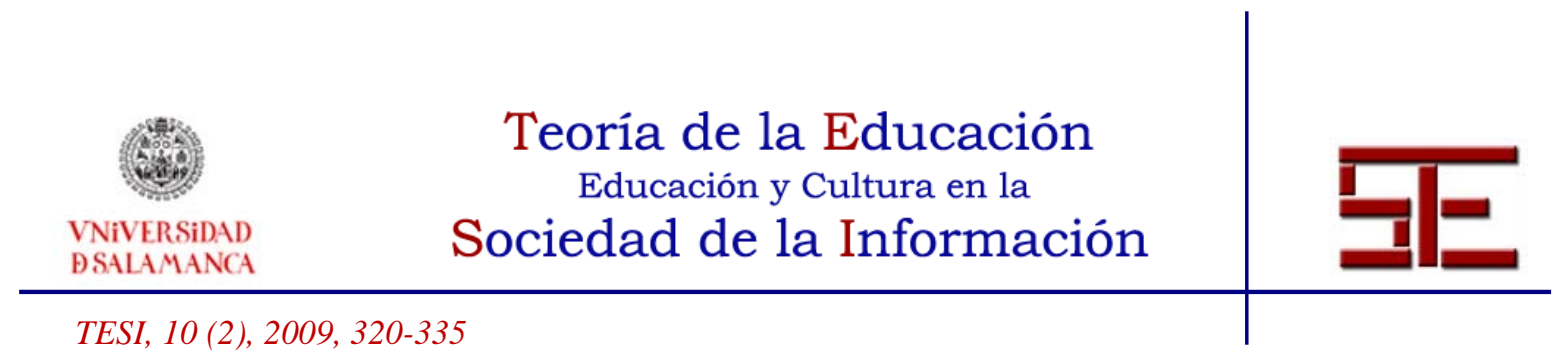

\title{
EDUCACIÓN EN VALORES Y EDUCACIÓN EMOCIONAL: PROPUESTAS PARA LA ACCIÓN PEDAGÓGICA.
}

Resumen: Relacionar la educación en valores con la educación emocional supone enfatizar la idea de que la educación consiste en ofrecer respuestas desde la institución escolar a todas las dimensiones de la persona: cognitivas, conductuales y afectivas -el pensar, el hacer y el sentir-. Respuestas que parecen no se han dado en sistemas educativos anteriores.

En este artículo planteamos la necesidad de rescatar una educación en valores que no sólo no olvide la educación emocional, sino que la explicite a través de programas de acción pedagógica cuyos objetivos se concreten en la construcción de la personalidad ética, la educación de los sentimientos y la competencia comunicacional, la adquisición de competencias éticas, la aceptación de límites y normas de convivencia, así como la gestión de las emociones y su consecuencia conductual: la autorregulación del comportamiento. La sensibilización y formación del profesorado resultará indispensable para se plantee y desarrolle en las instituciones educativas esta innovación.

Palabras clave: educación en valores, educación emocional, dimensiones de la persona, programas de acción pedagógica, competencias éticas

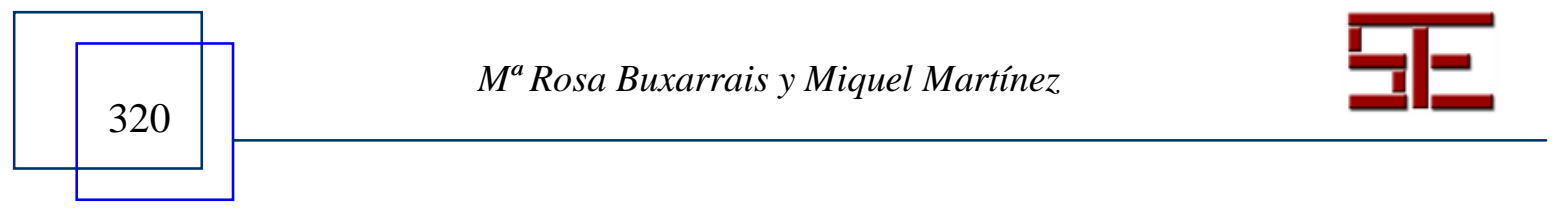




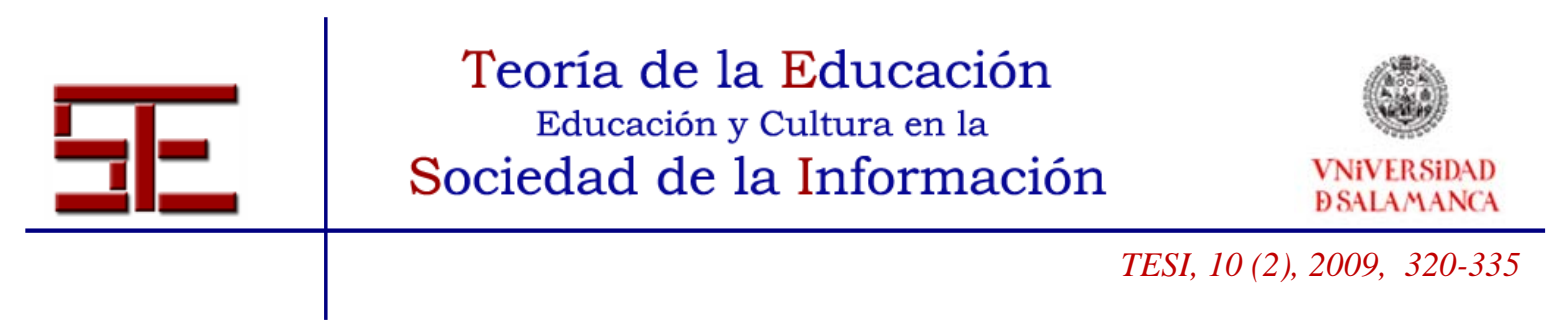

\section{ÉDUCATION AUX VALEURS ET ÉDUCATION ÉMOTIONNELLE: PROPOSITIONS POUR UNE ACTION PÉDAGOGIQUE.}

Résumé: Établir une relation entre l'éducation aux valeurs et l'éducation émotionnelle suppose emphatiser l'idée que Éducation signifie donner un réponse de la part de l'institution scolaire à toutes les dimensions de la personne : cognitives, conductuelles et affectives - penser, faire et sentir- des réponses qui semblent ne pas avoir été données par les systèmes éducatifs antérieurs.

Dans cet article nous envisageons la nécessité de récupérer une éducation aux valeurs qui n'oublie pas l'éducation émotionnelle si non qu'elle soit explicitée au travers des programmes d'action pédagogique dans lesquels les objectifs se concrètent dans la construction de la personnalité éthique, l'éducation des sentiments et la compétence communicationnelle, l'acquisition des compétences éthiques, l'acceptation des limites et normes de cohabitation ainsi que la gestion des émotions et sa conséquence conductuelle : l'autorégulation du comportement. La sensibilisation et la formation des professeurs seront indispensables pour pouvoir envisager et développer dans les institutions éducatives cette innovation éducative.

Mots clée: l'éducation aux valeurs, l'éducation émotionnelle, dimensions de la personne, programmes d'action pédagogique, acquisition des compétences éthiques

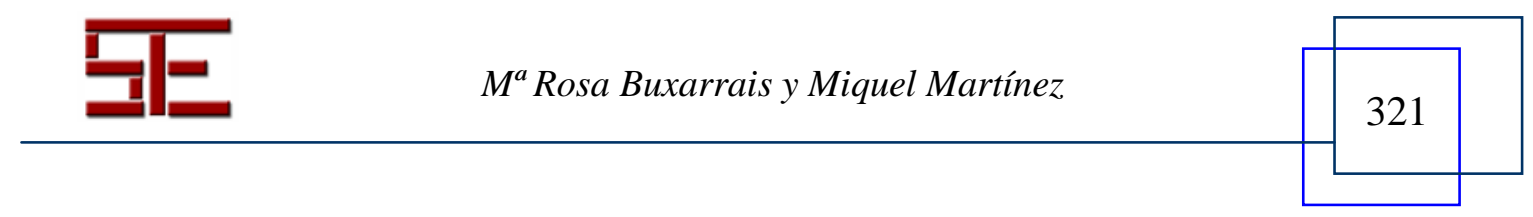




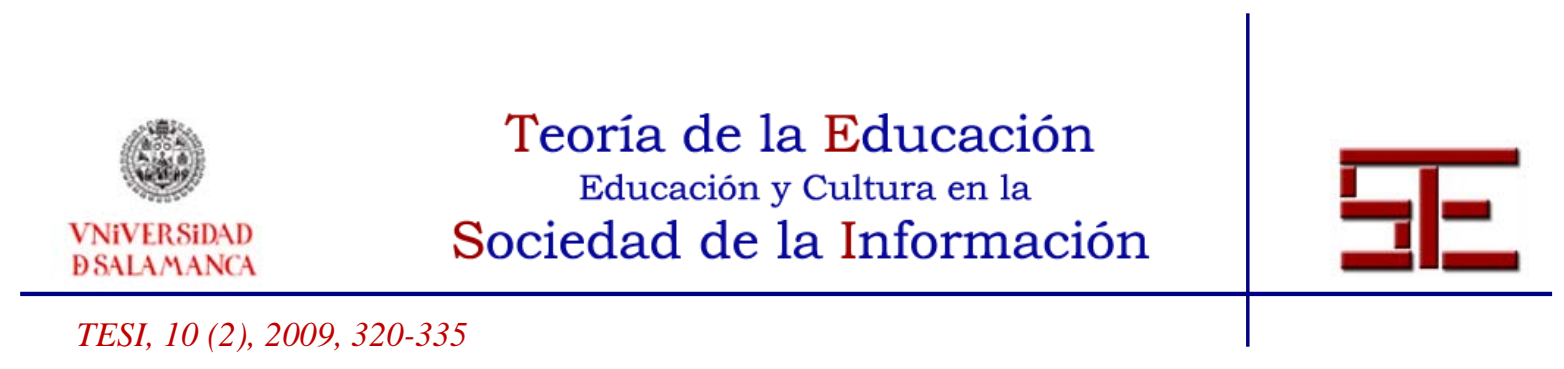

\section{EDUCATION IN VALUES AND EMOTIONAL EDUCATION: PROPOSALS FOR THE PEDAGOGICAL ACTION.}

Abstract: To relate the education in values with emotional education supposes to emphasize the idea that the education consists in offering answers from the educational institution to all the dimensions of the person: cognitive, behavioural and affectivethinking, doing and feeling -, answers that seems not have been given in previous educational systems.

In this article we raised the necessity to rescue an education in values that does not only forget the emotional education but it is also specified through programs of pedagogical whose objectives take shape in the construction of ethical personality, the education of feelings and the communicational competition, the acquisition of ethical competitions, the acceptance of limits and coexistence norms as well as the management of the emotions and its conductible consequence: self-regulation of the behaviour. The sensibility and training of the teaching staff will be indispensable so that this educational innovation could be considered and developed in the educational institutions.

Key words: education in values, emotional education, dimensions of the person, programs of pedagogical actions, acquisition of ethical competitions.

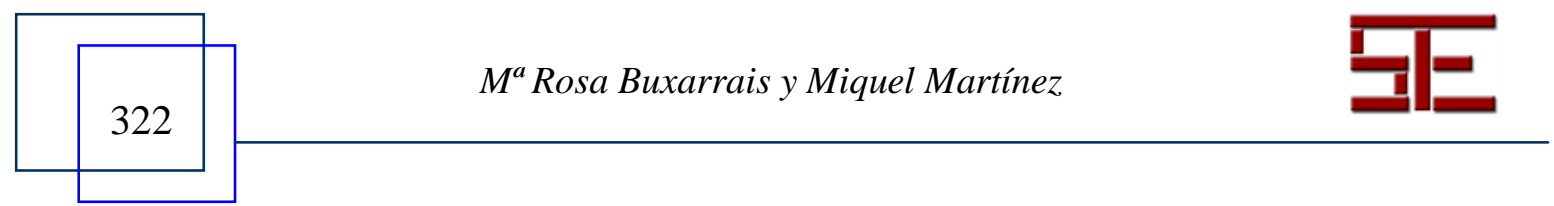




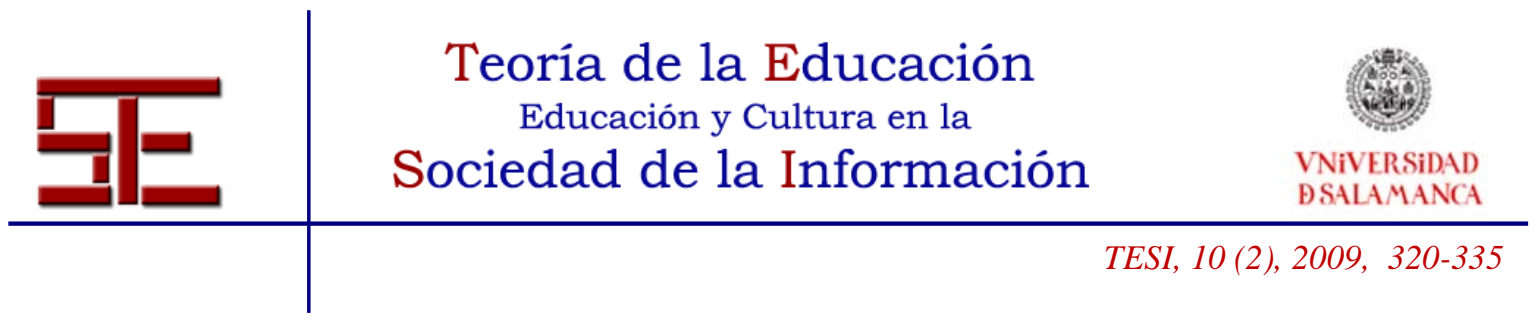

\section{EDUCACIÓN EN VALORES Y EDUCACIÓN EMOCIONAL: PROPUESTAS PARA LA ACCIÓN PEDAGÓGICA.}

\section{María Rosa Buxarrais Estrada \\ mrbuxarrais@ub.edu \\ Universidad de Barcelona. \\ Miquel Martínez Martín \\ miquelmartinez@ub.edu \\ GREM (Grup de Recerca en Educació Moral). \\ 1.- LA EDUCACIÓN EN VALORES: UNA EDUCACIÓN EMOCIONAL Y DE LOS SENTIMIENTOS SISTEMÁTICA.}

La pedagogía, en tanto que cuerpo de conocimientos sobre la educación, no ha prestado la debida atención al mundo de los sentimientos. Si bien es cierto que a lo largo de su historia las referencias a la importancia de la educación de los sentimientos están presentes, no lo es menos que la construcción de conocimiento en torno a tal educación, es decir, a cómo planificar y regular situaciones educativas con el objetivo intencional de optimizar las dimensiones de la persona relacionadas con el mundo de las emociones, los afectos y los sentimientos, no ha sido ni es aún hoy en día suficiente.

A pesar de las declaraciones de intenciones sobre la relevancia del tema, todavía queda mucho por hacer para incorporarlo de forma sistemática y oportuna en el discurso pedagógico, y mucho por saber para poder abordar la educación de forma integral. Educación integral que sólo es posible si contemplamos, junto al mundo de la razón, el mundo de las emociones y los sentimientos y el mundo de la voluntad. Este último, el mundo de la voluntad, relacionado con la gestión de las emociones y las formas de afrontar las situaciones vitales y la toma de decisiones, es también un mundo olvidado que conviene y urge recuperar para saber más sobre él y sobre cómo abordar mejor su educación.

Nuestra sociedad, la sociedad de la información, la diversidad y la globalización, favorece escasamente el mundo de la razón. Por ello, la educación no puede ni debe abandonar su cultivo y la pedagogía debe saber integrar los avances que, gracias a la filosofía, la psicología, la lingüística y otras disciplinas afines, nos permiten conocer mejor los procesos cognitivo-racionales. Sin embargo, no es suficiente con integrar tales

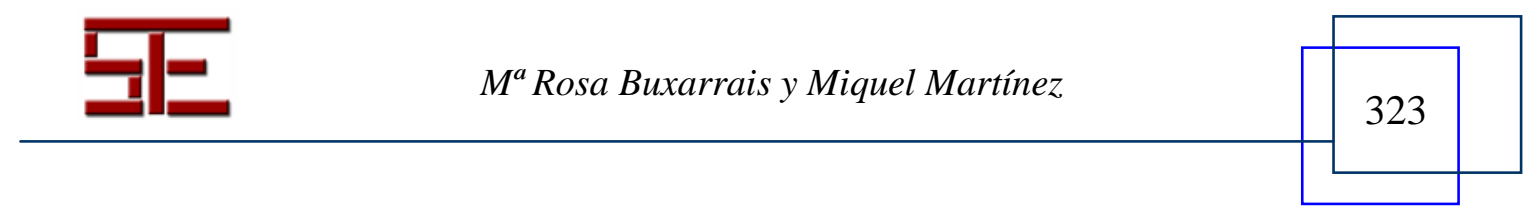




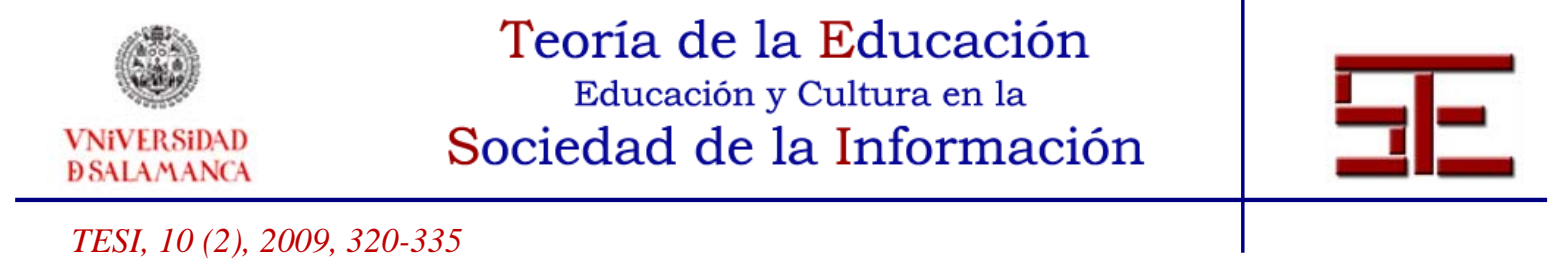

desarrollos y conocimientos, es necesario hacer lo mismo con los que se refieren al mundo de la voluntad y al de los sentimientos.

El aprendizaje humano no puede explicarse sin el concurso de estos tres mundos. Éstos no deben concebirse como partes separadas y susceptibles de estudio especializado de forma individual. Deben concebirse como integrantes de la acción humana en su acepción más amplia y de la interacción social y comunicativa que, como sujetos, mantenemos con el medio y los otros. Por ello, al referirnos a la educación de la persona no podemos considerar en ella zonas duras y zonas blandas, las primeras merecedoras dignas de construcción de conocimiento, es decir de investigación, y las segundas, zonas borrosas que debido a la dificultad que su comprensión nos plantea son analizadas y consideradas como fuente de pautas pedagógicas y educativas de una manera ingenua $\mathrm{y}$ con escaso fundamento.

Existe una concepción ingenua sobre la educabilidad de las personas en los términos de emociones, afectos y sentimientos que dificulta la acción educativa derivada del saber pedagógico. Afortunadamente los educadores, maestros y profesores, no esperan a que el saber pedagógico o psicológico avance para decidirse a actuar. La urgencia y viveza de su actividad les obliga a actuar no sólo en función del conocimiento científico acumulado y debidamente contrastado, sino también del derivado de la experiencia, la intuición y la imitación. Es en este segundo ámbito del conocimiento en el que frecuentemente se encuentra el saber relacionado con nuestro tema. Conviene no perder estas fuentes del saber, pero también completarlas con más conocimiento acumulado, sistemático y contrastado, si cabe, sobre el mismo.

Esta ignorancia pedagógica sobre el mundo de los sentimientos hace que, a pesar de que en teoría se formulen propuestas de acción pedagógica orientadas al desarrollo de modelos de educación integral, en la praxis se constaten ciertas dificultades para su logro. En algunos casos se constata cómo la práctica se centra excesivamente en el área cognitiva y conductual y, en otros, se observa una separación excesiva entre el área afectiva y el área cognitiva, lo que impide el tratamiento global de la persona.

Además, las respuestas a la realidad de nuestra sociedad, compleja y plural, exigen un nivel de comprensión, respeto y tolerancia difícil de alcanzar si ignoramos la importancia del mundo de la afectividad y de la sensibilidad en la vida de la relación y la comunicación (García Carrasco y García del Dujo, 2001). El carácter relacional y comunicacional presente en los tres tipos de prácticas educativas -formales, no formales

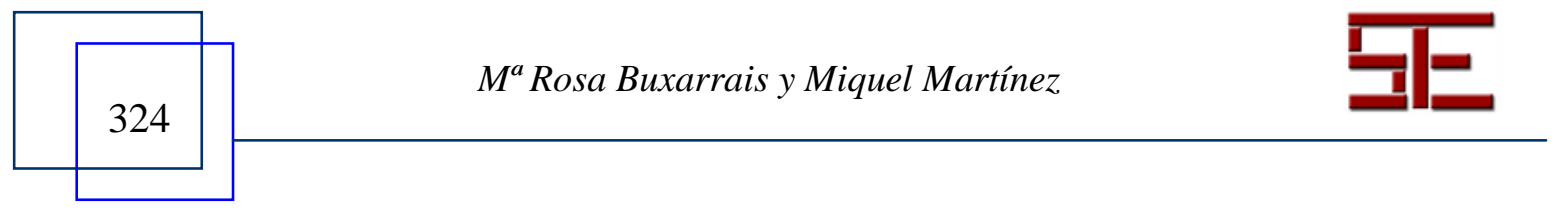




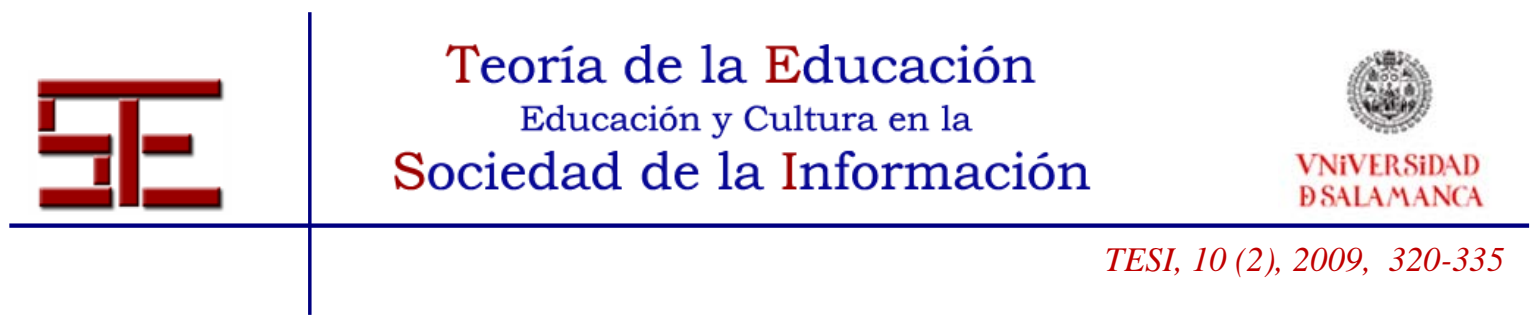

e informales-, hace que en todas ellas la afectividad y el mundo de la sensibilidad y de los sentimientos adquieran especial relevancia. La capacidad para comprender al otro antes de aceptarlo o no, y nuestra especial disposición genuinamente humana para captar su tono anímico son dimensiones de la persona que sólo pueden desarrollarse de forma completa mediante la educación de los sentimientos. Desde nuestra perspectiva, la importancia que otorgamos a las relaciones proviene de la concepción de la personan como sistema, organizado internamente, pero susceptible de modificación a partir de la retroalimentación fruto de la interacción constante con otros sistemas. Un sistema con la capacidad de conseguir niveles progresivos de autorregulación, gracias a la cual somos capaces de gobernar nuestra propia vida, relacionarnos con los otros y también de implicarnos en la transformación del entorno (Sanvisens, 1984; Martínez, 1986). Y es precisamente por la importancia que concedemos al carácter relacional de la educación, y en particular de la educación en valores, que afirmamos la necesidad de prestar una especial atención a las emociones y a los sentimientos debido a que éstos influyen en la persona no sólo a nivel intrapersonal, sino también interpersonal. Inciden en la regulación de nuestra conducta y en la configuración de nuestras respuestas a la realidad.

Pero además existen otros factores no sólo de carácter pedagógico, sino también sociales que obligan a abordar el mundo de las emociones y la educación de los sentimientos de forma más completa y sistemática que hasta ahora. Entre otros, podemos considerar el incremento progresivo de violencia en las escuelas y en las calles, la aparición de brotes de racismo ante el fenómeno desbordante de la inmigración, la emergencia de movimientos integristas, la falta de respeto en las relaciones interpersonales, la violencia doméstica, etc. Puede que éstas y otras causas hayan hecho saltar las alarmas de educadores, familias y sociedad en general, espectadores y actores de una sociedad donde se pretende conseguir la felicidad sin asumir responsabilidades evitando todo aquello que implique esfuerzo o sacrificio, donde no se valoran las normas como garantía de confianza mutua y de logro del bien común, donde se piensa en la satisfacción de las necesidades individuales antes que en las colectivas y en la que el respeto a los demás se ha convertido en un valor escaso.

Todas estas circunstancias nos obligan a plantear una educación en valores que no solamente no olvide la educación emocional, sino que la tenga en cuenta de forma explícita. Una educación de los sentimientos y de la dimensión social de la persona que contribuya a mejorar la convivencia entre las personas y, como consecuencia, la sociedad. En este sentido, una de las competencias que se nos plantea en el documento

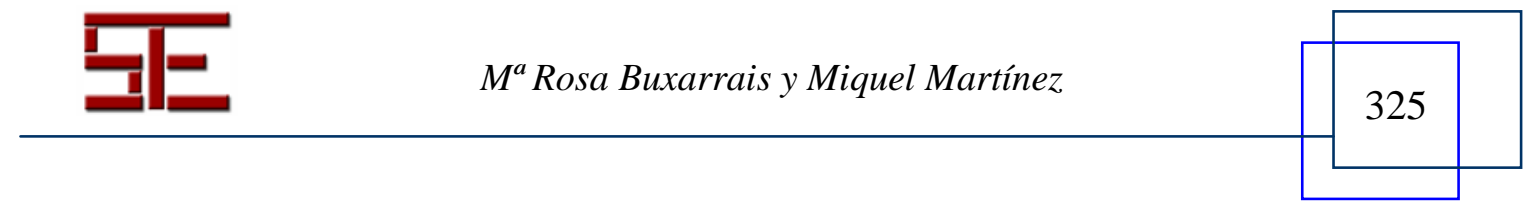




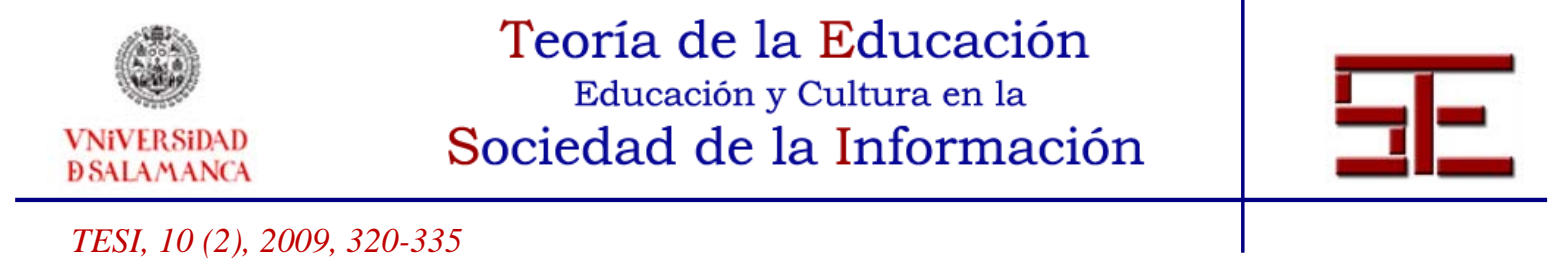

DeSeCo (Proyecto de la Organización para la Cooperación y el Desarrollo Económicos) está relacionada con esta dimensión: el actuar en grupos socialmente heterogéneos que suponga saber convivir y contribuir a la obtención de comunidades guiadas por criterios de justicia y felicidad.

Es necesaria la promoción de la sensibilidad, una de las dimensiones indispensables para la interiorización de valores como el respeto, la solidaridad y la justicia. Desde la psicología se utiliza el término empatía (Hoffman, 2002), que se define como la capacidad que facilita el ponerse en el lugar del otro, ya que la progresiva descentración posibilita el conocimiento y la comprensión de las razones, sentimientos, actitudes y valores de las demás personas. Por ello conviene recuperar y destacar el valor de la compasión, entendiendo ésta de acuerdo con Colby y Damon (Colby y Damon, 1992) según afirman en Some do care, como: 1) un compromiso permanente con principios morales que incluyen un respeto generalizado por la humanidad o una evidencia de virtud moral; 2) una disposición para actuar de acuerdo con los principios morales, lo que implica una consistencia entre las acciones y las intenciones, los medios y los fines; 3) la voluntad para arriesgar los intereses personales en beneficio de los valores morales; 4) una tendencia a inspirar a otras personas para la acción moral (una capacidad para contagiar su visión del mundo y su acción de ayuda); 5) humildad en torno a su importancia y una despreocupación por su ego. El concepto de "care” se sitúa en otra órbita, la de la entrega, la disponibilidad y la donación gratuitas, y nos permite insistir en las interconexiones entre individuos y la naturaleza de la coexistencia humana (Jollonch, 2006). Dicho concepto debe retomarse para convertirlo en propuesta para la acción pedagógica.

\section{2.- LA CONSTRUCCIÓN DE LA PERSONALIDAD ÉTICA.}

Obviamente los sentimientos nos ayudan en la construcción de nuestra propia biografía, a establecer un vínculo (Taylor, 2002) afectivo eficaz y, a la vez, nos permiten mantener una organización jerarquizada y flexible de nuestra matriz de valores. Es la totalidad del cuerpo la que se involucra en la incorporación de una experiencia y en la comunicación de la experiencia (García Carrasco, 2003, 83). Sentimientos, acción y razón se integran mutuamente y nos sirven para construir dicha matriz. Así, comunicarse con sensibilidad con otras personas supone potenciar buenas relaciones interpersonales, facilitando la gestión de conflictos, discusiones, persuasión y negociación, elementos previos al desarrollo de la escucha activa, de una actitud dialógica y empática (Puig, 2003;

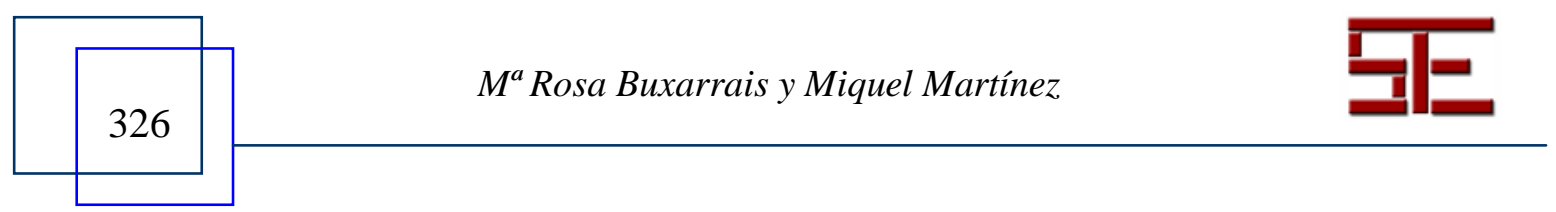




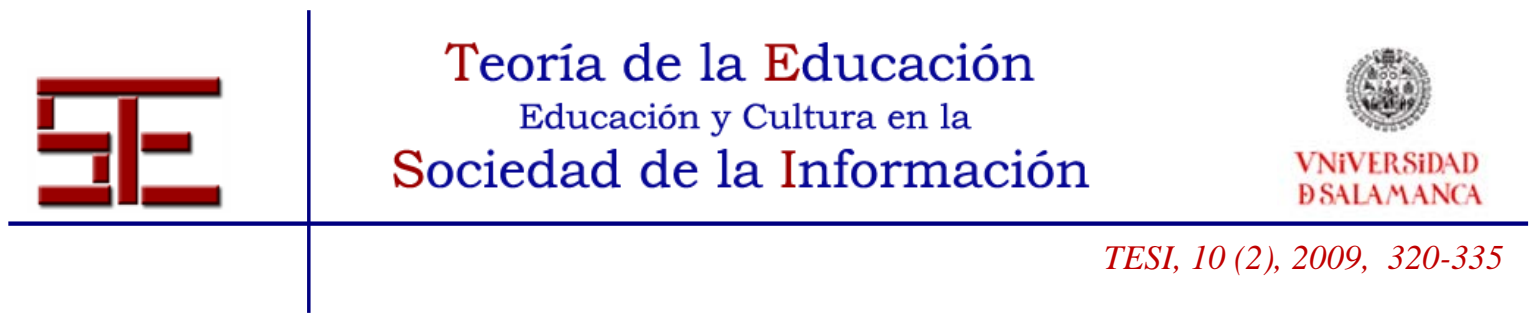

Martínez \& Bujons, 2001) fundamental para actuar en contextos pluralistas y democráticos.

La persona en sus manifestaciones combina sus diferentes dimensiones entrelazadas y yuxtapuestas de forma que constituyen un sistema y como tal funciona. Nos estamos refiriendo a las dimensiones: cognitiva-racional, afectiva-emocional y volitivaconductal. Resulta imposible identificar separadamente cuál de estas dimensiones se pone en marcha cuando la persona se encuentra en una situación de interacción social, y es sujeto de educación y objeto de la misma. Cuando educamos estamos incidiendo en cada una de estas dimensiones, en mayor o menor medida, con el objetivo de contribuir a la optimización integral de la persona.

La educación en general, y la educación en valores en particular, movilizan un conjunto de procesos que desembocan en el aprendizaje ético de los educandos. Este tipo de aprendizaje es promovido por diversos agentes y mediante diferentes vías (Martínez \& Bujons, 2001). La institución educativa es uno de estos agentes y, probablemente, uno de los más potentes. Sin embargo, no es el único. Sus efectos pueden ser y son contrarrestados por la intervención de otros agentes cuya intencionalidad educativa se cuestiona. Nos referimos a agentes de carácter informal y con mayor poder de influencia. Entre éstos consideramos a la familia, los medios de comunicación -la televisión y las pantallas-, los agentes sociales y culturales y, por supuesto, los iguales, con los que convivimos en el mundo del ocio y del trabajo. Todos ellos, la escuela también, son buenos escenarios para aprender valores y contravalores, espacios en los que se viven y practican los valores y donde se observan y se construyen sistemas de valores propios y singulares.

En todos ellos se puede constatar cómo la construcción de valores parte del mundo de los sentimientos, de la comprensión de las situaciones y de los valores que caracterizan nuestra cultura y nuestro espacio vital -momento hermenéutico y de la sensibilidad moral-; y avanza hacia la propuesta de valores, principios y normas morales con pretensión de verdad, corrección y veracidad -momento discursivo- (Hoyos, Martínez, 2004). En este proceso, la educación de los sentimientos y el aprendizaje de competencias comunicacionales son clave y se convierten en objetivos educativos fundamentales.

No es nuestro propósito aquí hacer una relación exhaustiva de nuestra perspectiva sobre educación en valores o aprendizaje ético, de manera que solamente nos referiremos a las

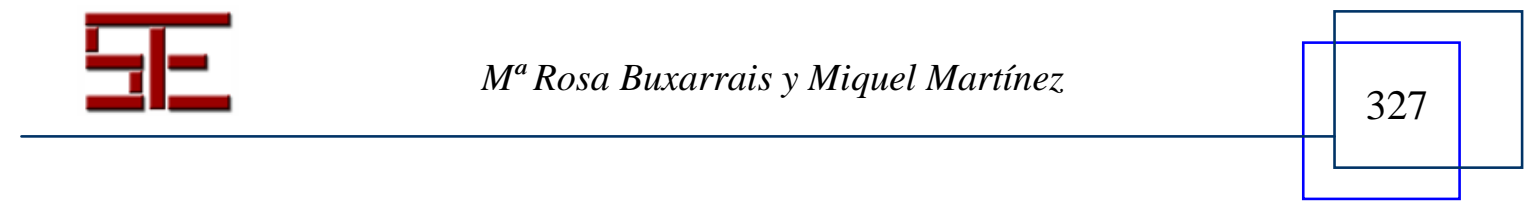




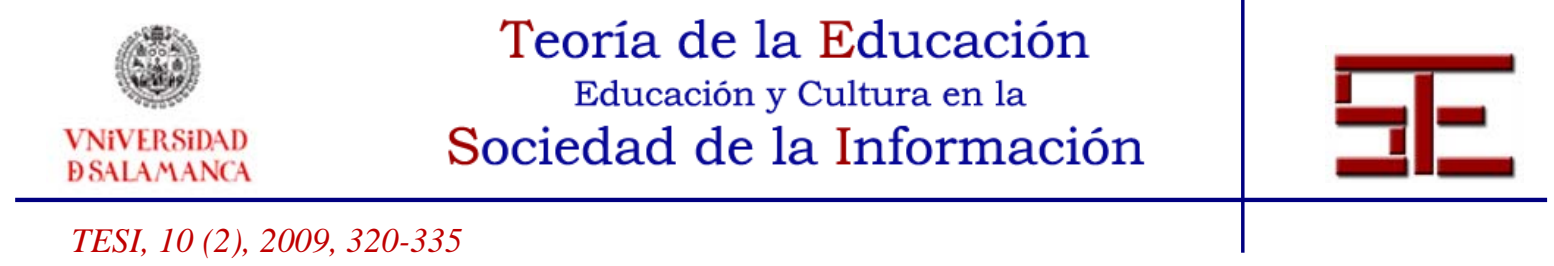

cuestiones más significativas que nos ayuden a entender la propuesta pedagógica que realizamos. Nuestra intención es promover aprendizajes éticos que permitan construir matrices singulares de valores guiadas por ideales de libertad, justicia, dignidad, compasión y responsabilidad. Son propuestas orientadas a la construcción de modelos de vida buena y feliz a nivel individual que, a su vez, promuevan modelos de vida justa y digna para todas las personas, en tanto que miembros de una comunidad.

\section{3.- EDUCAR EN VALORES EN DISTINTOS ESPACIOS EDUCATIVOS.}

Educar en valores es una tarea presente en distintos espacios educativos, formales e informales. En todos ellos, debe suponer el reconocimiento de la cultura y los sentimientos de las personas que intervienen. Esta cultura de la aceptación de los otros, antes incluso de que podamos comprenderlos, deviene en condición necesaria para poder educar y contribuir a la construcción autónoma de los sujetos que en ella participan.

Desde el espacio de la familia y de la educación no formal y hasta los espacios que requieren mayor planificación pedagógica, como por ejemplo la escuela, la educación en valores debe partir de la realidad próxima y de la cultura real de los sujetos que intervienen. Por ello la aceptación de la persona desde los primeros momentos y su reconocimiento como sujeto de derechos y de sentimientos y ponernos en su lugar para darnos cuenta de sus necesidades y de su sufrimiento son requisitos básicos para iniciar la tarea educativa.

Sin embargo este reconocimiento y aceptación deben estar acompañados de otros factores que permitan el crecimiento y la construcción de la persona. Aquí queremos destacar los dos siguientes:1) un entorno de aprendizaje y convivencia caracterizado por una atmósfera afectiva que integre variedad de estímulos y relaciones interpersonales que contribuyan a que cada persona se forme una imagen ajustada de sí mismo e incremente su autoestima y, 2) que la persona aprenda a aceptar y reconocer que existen unos esquemas de referencia que debe aprender a respetar. Se trata de ofrecerle unos límites que, sin ser inflexibles, sean suficientemente estables como para permitirle aprender que es sujeto de derechos, sentimientos y deberes. En este sentido conviene considerar (Payà, 1997, 195) que la consecución de una moral autónoma sin más, sin la consideración de la otra persona "con rostro" (Mèlich y otros, 2002), invalidaría todo proceso comunicativo y dialógico y no constituiría tampoco optimización y desarrollo

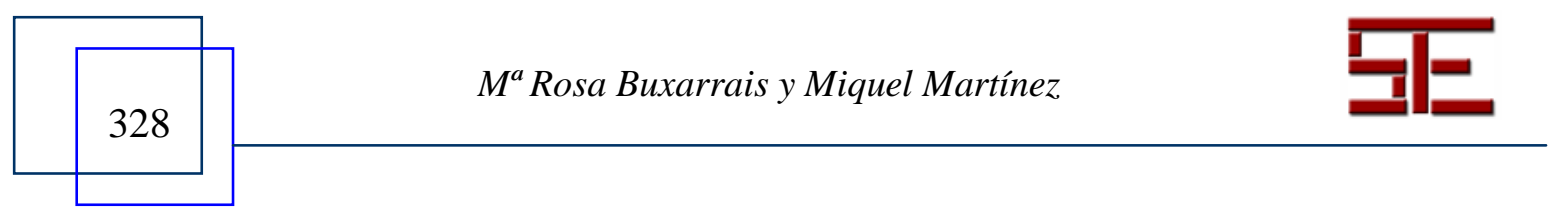




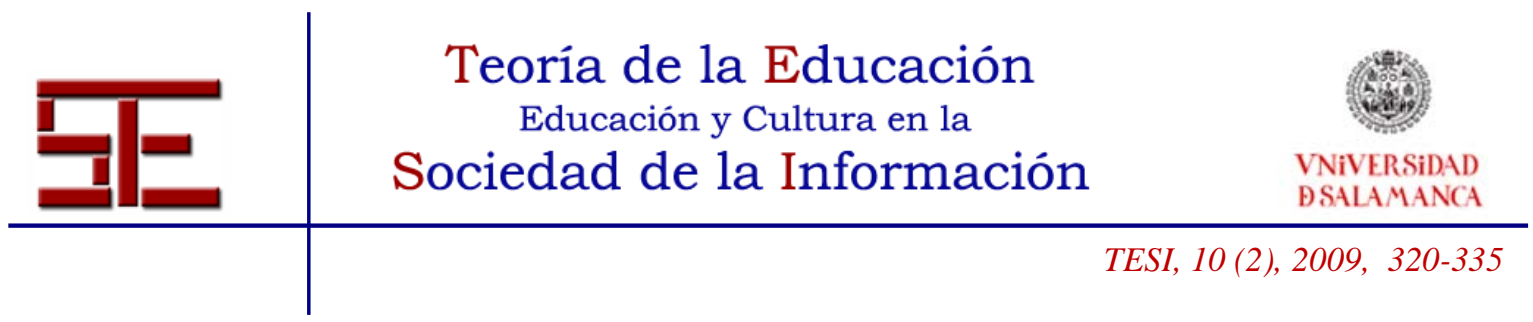

humano. El respeto a la dignidad de la otra persona permite descubrir y alcanzar en su plenitud el ideal de autonomía.

\section{4.- ADQUISICIÓN DE COMPETENCIAS ÉTICAS.}

Educar en valores supone contribuir a que la persona adquiera competencias para construir su sistema de valores y acepte y considere como buenos determinados ideales. No consiste sólo en formar personas autónomas ni, por supuesto, sólo personas que reconozcan y acepten su cultura y sus sentimientos, sino que sean competentes en tanto que ciudadanos y ciudadanas en sociedades caracterizadas por la diversidad.

Dirigirse hacia la educación de una ciudadanía activa en sociedades pluralistas y democráticas conlleva la promoción de aprendizajes éticos que contribuyan al logro de competencias que permitan a la persona comprender aquellas cuestiones socialmente controvertidas y moralmente relevantes para sí misma, y también para su comunidad.

Supone conocer sus derechos y deberes como ciudadanos y saber ejercerlos, responsabilizarse de sus acciones, renunciar al bien particular cuando dificulta el bien común, ponerse en la piel del "otro" para desarrollar la actitud de acogida a ese "otro” y ser capaz de construir un modelo de vida que combine el mundo de lo particular con algún criterio que permita la construcción de comunidad, de un mundo compartido, justo y solidario.

Como consecuencia, debemos plantearnos la educación en valores como la transmisión de contenidos informativos, procedimentales y de actitudes, valores y normas que supongan un aprendizaje que permita realizar un análisis crítico de nuestras culturas y de las de los demás y un avance comunicacional en la construcción de una cultura compartida.

\section{5.-EDUCAR LOS SENTIMIENTOS Y LA COMPETENCIA COMUNICACIONAL.}

Educar en valores de manera integral significa, además, educar los sentimientos, ya que éstos van de la mano de la razón, la argumentación y la competencia comunicacional de las personas. Las situaciones de aprendizaje dialógico en las que participamos y avanzamos discursivamente con los "otros", iguales o diferentes, nos ayudan a regular nuestros sentimientos y nuestra voluntad.

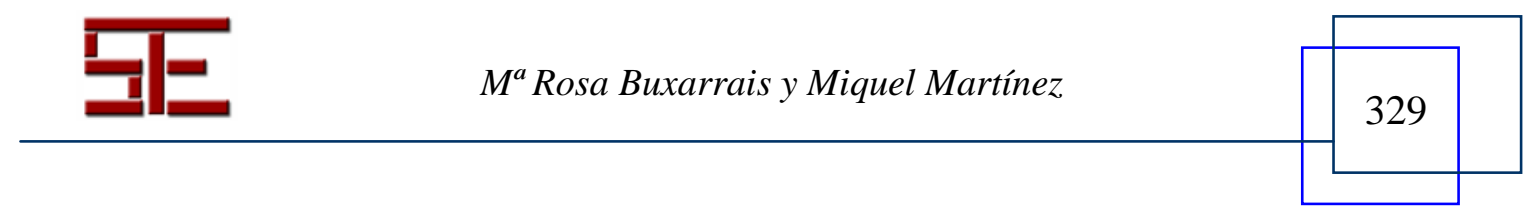




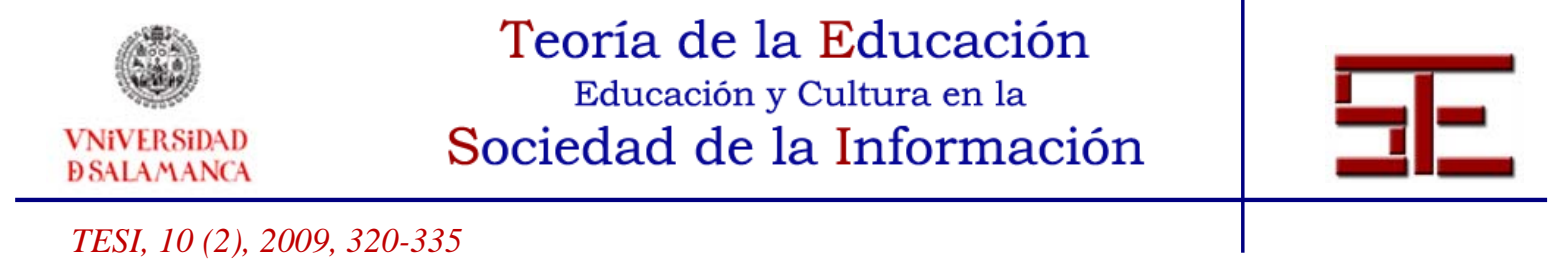

El aprendizaje y desarrollo de la competencia comunicacional y de criterios de racionalidad puede alcanzarse con mayor eficacia si promovemos situaciones de aprendizaje cooperativo en los que las personas que intervienen están agrupadas atendiendo a criterios de heterogeneidad en la forma de abordar y resolver situaciones de la vida cotidiana.

Las diferentes percepciones de la realidad y los sentimientos que cada sujeto construye a partir de las mismas son factores potenciales de conflicto sociocognitivo que mediante el uso del lenguaje pueden conducir desde una comprensión basada fundamentalmente en la sensibilidad moral a un nivel de comprensión elaborada discursivamente que permita construir y compartir leyes o principios generales que regulen el mundo de los valores.

Estas situaciones de aprendizaje cooperativo y dialógico implican ejercicios de autoconocimiento, comprensión de nuestros sentimientos, gestión de nuestras emociones, conocimiento y aceptación de los otros, sufrir con los otros. Son escenarios potencialmente óptimos para el aprendizaje mediante la práctica y la observación de valores como: el respeto, la diferencia de criterio, la solidaridad, la diversidad de sentimientos y formas de comprender la realidad y el diálogo. Asimismo, y siempre que el educador este formado adecuadamente, son buenos espacios en los que aprender a conocerse a sí mismo y a percibir que somos reconocidos por los demás y valorados, incrementar nuestra autoestima personal y competencia para atreverse a ser autónomo y establecer nuestras propias reglas.

\section{6.- ACEPTACIÓN DE LÍMITES Y NORMAS DE CONVIVENCIA.}

Nuestra civilización promueve estilos de vida y concepciones del mundo ajenas a la existencia de límites. Cada día crecen las desigualdades y aparecen nuevas formas de exclusión y vulnerabilidad social. Nos vemos asediados por imágenes desoladoras de un mundo que genera nuevas formas de "residuos humanos" (Bauman, 2004). Por eso, será necesario educar en la aceptación de límites y normas que regulen la convivencia.

En sociedades democráticas donde se supone que las normas son modificables, la educación en valores deberá procurar que las personas estén en condiciones para transformarlas y mejorarlas sin olvidar la promoción del apego a tales normas, porque son garantía de confianza activa entre los miembros de una comunidad en el logro del bien común. Por lo tanto, este tipo de educación debe afectar a los sentimientos. Porque la percepción de satisfacción o bienestar asociada a las conductas de apego a las normas

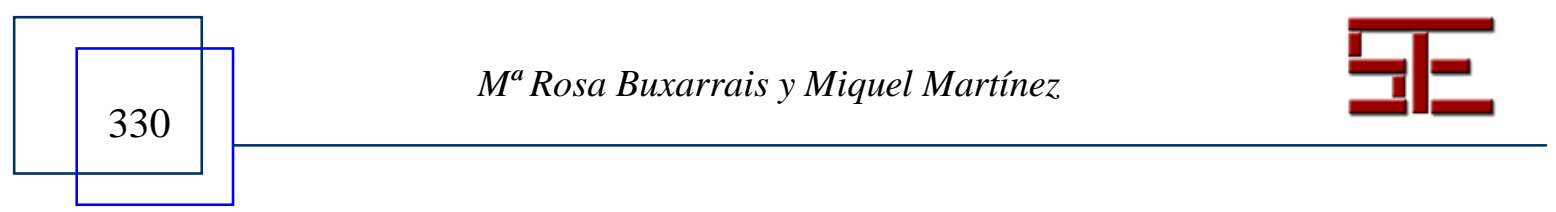




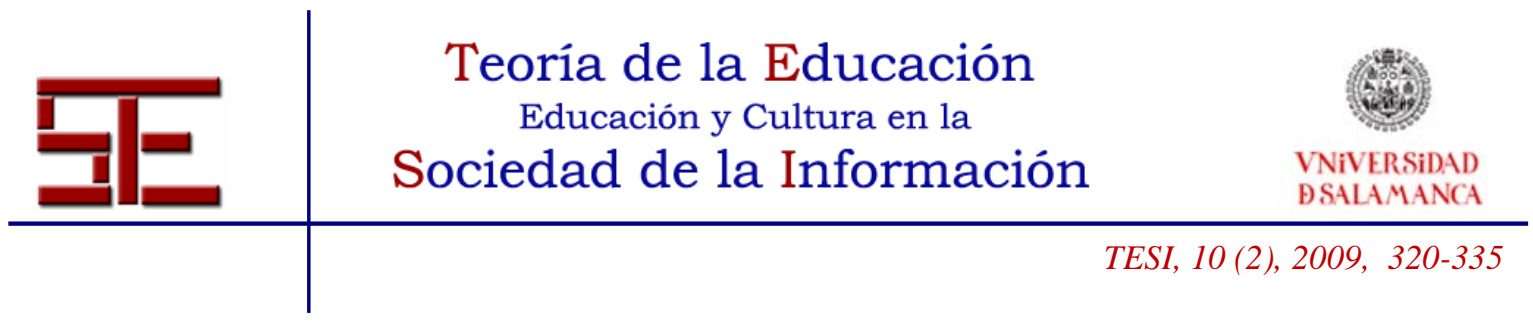

que regulan el bien común, en cuya modificación es posible participar, supone valorar positivamente el esfuerzo y la superación personal, no sólo en el caso de logro de bienes particulares, sino también en aquellos que afectan al conjunto de la comunidad.

Las personas, al igual que las civilizaciones, que no se conocen a sí mismas, que no conocen sus límites, son más vulnerables y, en situaciones de dificultad, pueden ser incompetentes para regular sus vidas de forma sostenible. Educar los sentimientos, pues, supondrá incorporar una educación en la aceptación de que las personas somos seres limitados.

\section{7.- GESTIÓN DE LAS EMOCIONES Y AUTORREGULACIÓN DEL COMPORTAMIENTO.}

Ser conscientes de nuestros sentimientos y aprender a gestionar las emociones que de ellos se derivan constituye una buena práctica para la autorregulación de nuestro comportamiento. Y también la disminución de nuestra vulnerabilidad ante los efectos manipuladores y adoctrinamiento que, desde posiciones contrarias a la promoción de la autonomía personal y la compresión crítica de la realidad, están proliferando en nuestra sociedad.

La oferta de estímulos y factores que acompañan al modelo de cultura asociado a la sociedad de consumo en la que vivimos ejercen un poder manipulador y conformador en las formas de entender el mundo, de identificar los problemas y sus causas y de entender la diversidad y sus consecuencias que no siempre son respetuosas con la libertad y la autonomía de la persona para elaborar su opinión. En estos procesos de aprendizaje social, la combinación de imagen, sonido y movimiento producen efectos emocionales que la persona debe saber regular, previa toma de conciencia de los mismos. Porque si no lo hace, se enfrenta a una vulnerabilidad alta ante los medios de comunicación que promocionan un determinado tipo de ocio y consumo. Y se encontrará indefensa ante mensajes que, manipulando sus estados emocionales, dificultan su competencia para comprender críticamente la realidad y actuar en consecuencia.

La educación de los sentimientos no solamente se dirige a aquellos sentimientos que se consideran deseables, positivos o ideales, sino que abarca toda clase de sentimientos. Todos los que aparecen en nuestra vida personal cuando interaccionamos con las demás personas. Por ejemplo, sentimientos que se derivan de la sensación de frustración que

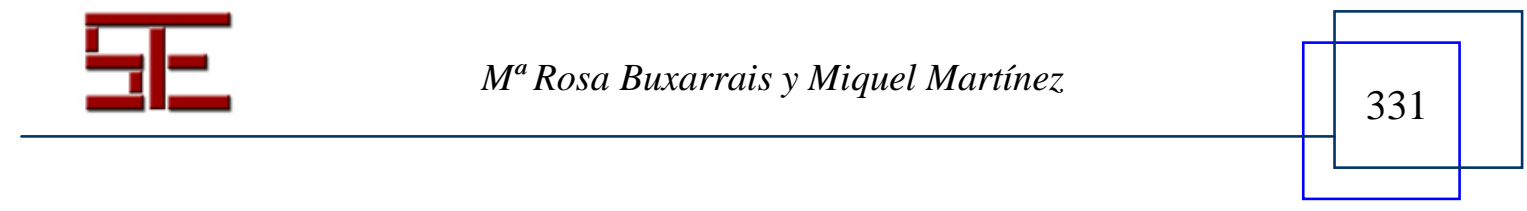




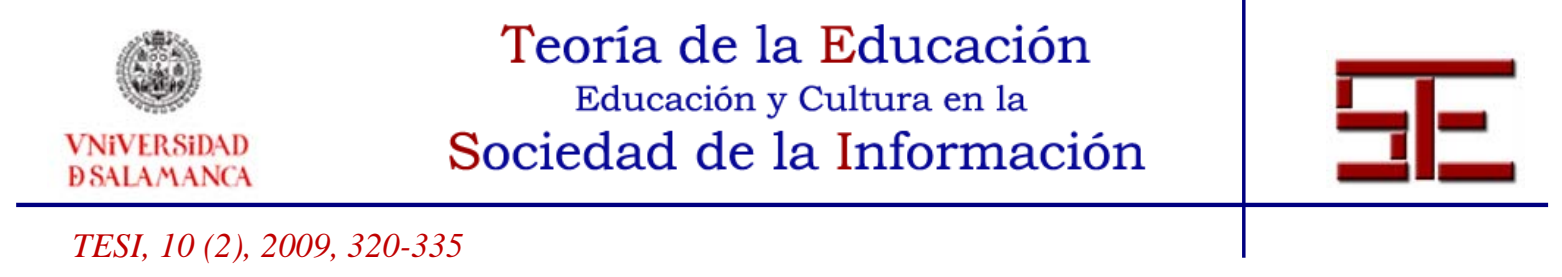

puede producirse cuando alguien en quien confiamos actúa de forma contraria a la que esperábamos, cuando contemplamos la violación de los derechos de las personas y nos genera indignación, cuando vemos que nuestras acciones tienen unas consecuencias que perjudican a los demás, etc. Este tipo de sentimientos a menudo son evitados por los educadores en el intento de hacer un bien al educando. Evitar que el educando viva estos sentimientos puede perjudicarle en cuanto que no le proporciona la oportunidad de practicar la gestión de las emociones y protagonizar su vida emocional de forma más completa.

En las situaciones que vivimos en la vida cotidiana a nivel de sentimientos, desempeñamos tres tipos de funciones según sea nuestra ubicación en las mismas: paciente, observador y agente. Es necesario que desde la educación se planteen pedagógicamente situaciones donde se puedan jugar cada uno de esos roles.

\section{8.- A MODO DE CONCLUSIÓN}

La educación de los sentimientos y la educación en valores no pueden ser fruto de la improvisación y la buena voluntad de los educadores. Éstas deben estar sujetas a una intencionalidad y sistematicidad de propuestas para que lleguen a buen puerto. Una formación inicial y continua de los profesionales de la educación, y en particular del profesorado; la posibilidad real de desarrollar proyectos educativos que integren estas cuestiones y que estén elaborados y compartidos por equipos docentes; y la sensibilización de todos los actores de la comunidad educativa son indispensables para que este tipo de cuestiones se planteen y desarrollen en las instituciones educativas y en las condiciones necesarias para el logro de lo que hemos venido planteando hasta aquí.

\section{BIBLIOGRAFÍA}

Berkowitz, M.W. (1995). Educar a la persona moral en su totalidad. En Revista Iberoamericana de Educación, nº 8, 73-101.

Buxarrais, M.R. (1997). La formación del profesorado en educación en valores. Bilbao: Desclée de Brouwer.

Buxarrais, M.R. (2000). Tendencias y modelos de educación moral. En Diálogo Filosófico, no $47,196-220$.

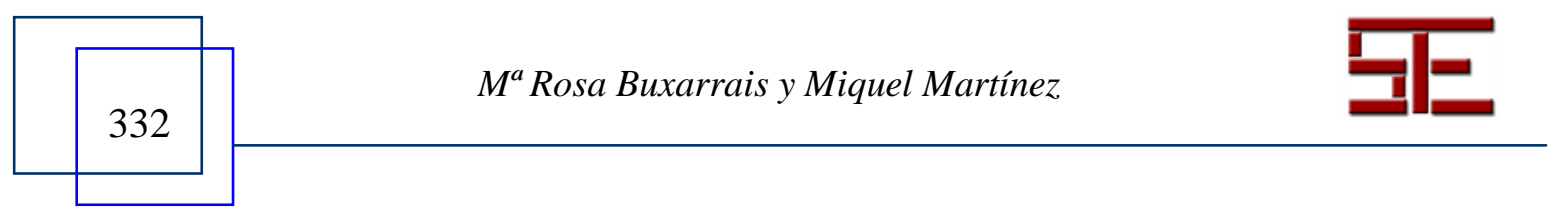




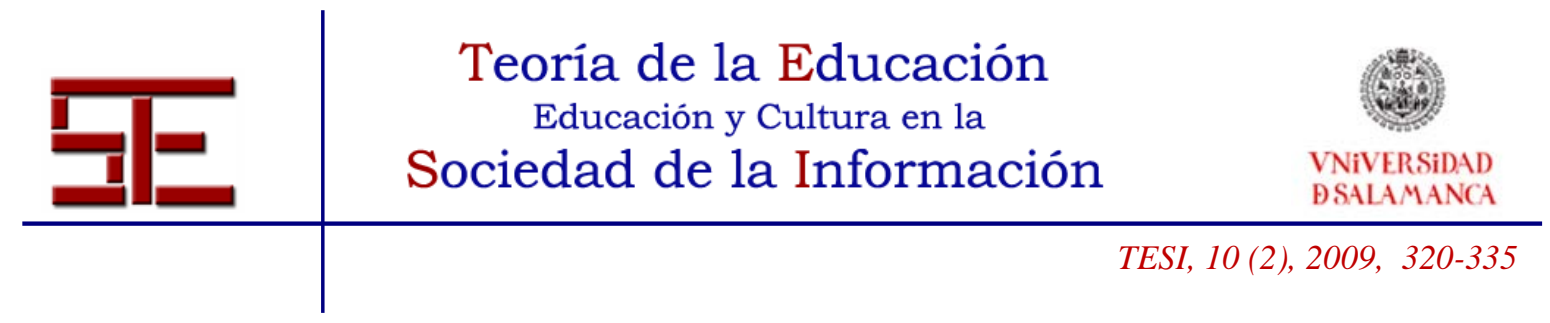

Buxarrais, M.R. y Martínez, M. (2004). «Children’s Rights in an Age of Information and Communication Technologies». Encounters on Education, vol. 5, pp. 141-159.

Cardús, S. (2000). El desconcert de l'educació. Barcelona: La Campana.

Chomsky, N. (2001). La (Des)educación. Barcelona: Crítica.

Colby, A., y Damon, W. (1992). Some do care. Contemporary lives of moral commitment. New York, Free Press.

DeSeCo: http://www.portal-stat.admin.ch/deseco/news.htm.

García Carrasco, J.; García del Dujo, A. (2001). Teoría de la Educación II. Procesos primarios de formación del pensamiento y la acción. Salamanca: Ediciones Universidad de Salamanca.

Garcia Carrasco, J. (2003). Evolución del contexto social y cambios en el pensamiento sobre la educación. Educación por la influencia o educación desde la experiencia. En AA.VV. Teoría de la Educación, ayer y hoy. Murcia: SITE.

Gilligan, C. (1985). La moral y la teoría. Psicología del desarrollo moral femenino. México, FCE.

Hansen, T. (2002). Explorando el corazón moral de la enseñanza. Barcelona, Idea Books.

Hoffman, M.L. (2002). Desarrollo moral y empatía. Barcelona: Idea Books.

Hoyos, G. y Martinez, M. (coords) (2004). ¿Qué significa educar en valores hoy? Madrid, Octaedro.

Jolonch, A. (2006). La vulnerabilitat, un horitzó per l'educació? En CASTIÑEIRA, A., y CALSINA, M. (coords.). Catalunya, reptes ètics. Barcelona: Proa.

Katz, M.S., Noddings, N. y Strike, K.A. (2002). Justícia y cuidado. En busca de una base ética común en educación. Barcelona, Idea Books.

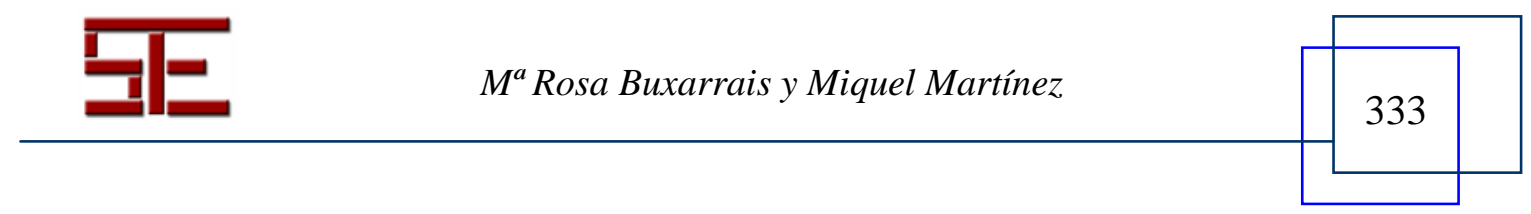




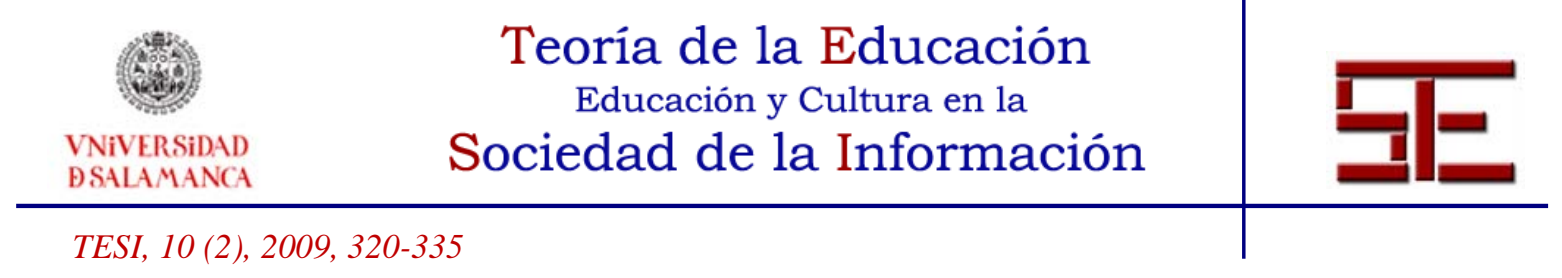

Kung, H. (1992). Proyecto de una ética mundial. Madrid, Trotta.

Martínez, M. (1986). Inteligencia y educación. Barcelona: PPU.

Martinez, M. (1998). El contrato moral del profesorado. Condiciones para una nueva escuela. Bilbao: Desclée de Brouwer.

Martinez, M., y Bujons, C. (coords) (2001). Un lugar llamado escuela en la sociedad de la información y la diversidad. Barcelona, Ariel.

Martinez, M., Puig, J.M. y Trilla, J. (2003). Escuela, profesorado y educación moral. En Revista de Teoría de la Educación. Salamanca, Ediciones Universidad, Vol. 15. 57-94.

Martínez, M. y Hoyos, G. (coords) (2006). La formación en valores en sociedades democráticas. Barcelona, Octaedro-OEI.

Mèlich, J.C., y otros (2000). La veu de l'altre. Reflexions i experiències per educar en valors ètics. Barelona, ICE-UAB.

Morin, E. (1999). Los siete saberes necesarios para la educación del futuro. Barcelona: Paidòs.

Nussbaum, M.C. (2002). El cultivo de la humanidad. Barcelona: Editorial Andrés Bello.

Naval, C. (2001). Confiar: cuna de la sociabilidad humana. San José de Costa Rica, Promesa.

Noddings, N. (2002). Educating moral people: a caring alternative to character education. Nueva York, Teachers College Press.

Ortega, P., y Minguez, R. (1999). The Role of Compasión in Moral Education. Journal of Moral Education. 28, 5-17.

Ortega, P., y Minguez, R. (2001). La educación moral del ciudadano de hoy. Barcelona, Paidós.

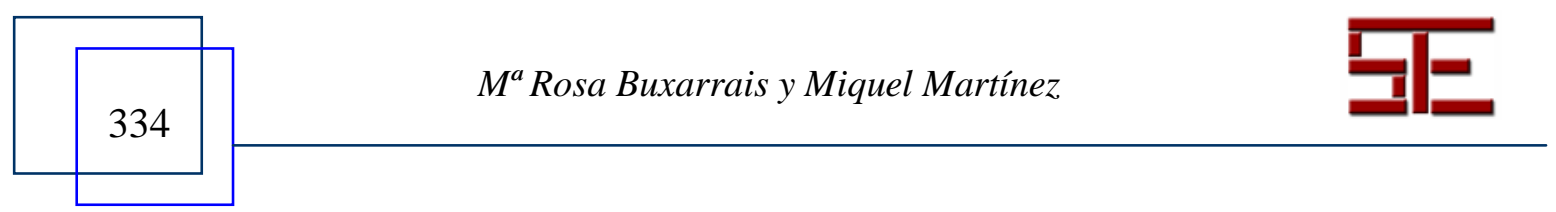




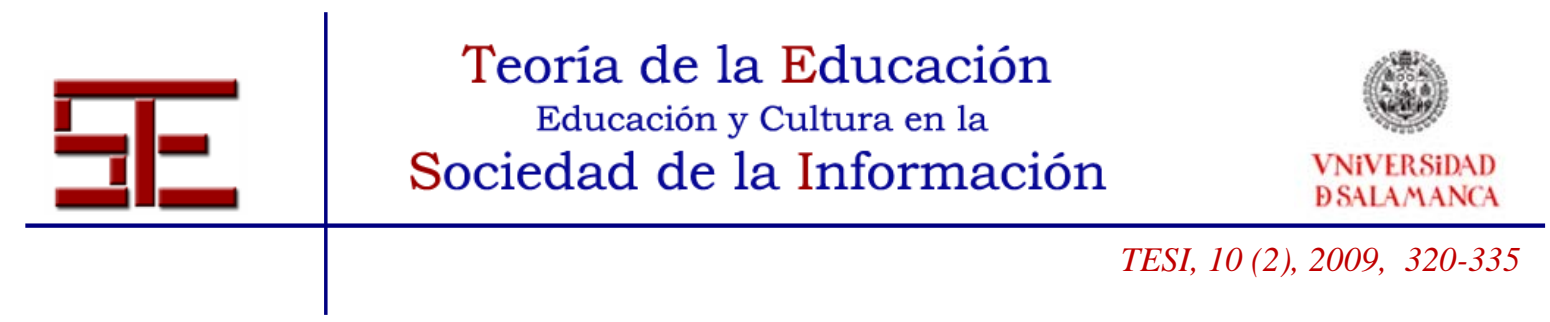

Paya, M. (1997). Educación en valores para una sociedad abierta y plural. Bilbao, Desclée de Brouwer.

Puig, J.M. (2003). Prácticas morales. Una aproximación a la educación moral. Barcelona, Paidós.

Puig, J.M. y otros (2006). Aprenentatge-servei. Educar per a la ciutadania. Barcelona: Octaedro.

Sanvisens, A. (1984). La cibernética de lo humano. Barcelona: Oikos-Tau.

Taylor, S.E. (2002). Lazos vitales. De cómo el cuidado y el afecto son esenciales para nuestras vidas. Barcelona, Taurus.

Tey, A. y Martinez, M. (2003). Educar en valors és educar en sentiments morals. En Educar, 31, 11-32.

Van Manen, M. (1998). El tacto en la enseñanza. El significado de la sensibilidad pedagógica. Barcelona, Paidós Educador.

Para citar la presente editorial puede utilizar la siguiente referencia:

Buxarrais, Ma R. y Martínez, M. (2009). Educación en valores y educación emocional: propuestas para la acción pedagógica, en Ortega Sánchez, I. y Ferrás Sexto, C. (Coord.) Alfabetización Tecnológica y desarrollo regional. Revista Electrónica Teoría de la Educación: Educación y Cultura en la Sociedad de la Información. Vol. 10, n ${ }^{0} 2$. Universidad de Salamanca, pp. 320-335 [Fecha de consulta: dd/mm/aaaa].

http://campus.usal.es/ revistas_trabajo/index.php/revistatesi/article/view/7519/7552

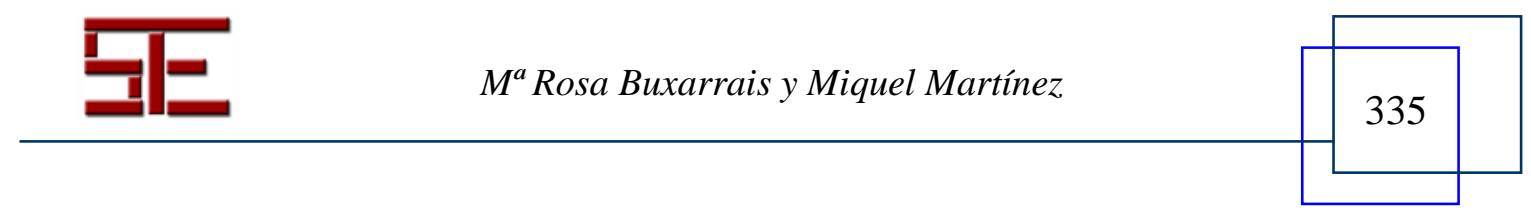

